\title{
Physician mothers' experience of workplace discrimination: a qualitative analysis
}

\author{
Meghan C Halley, ${ }^{1,2}$ Alison S Rustagi, ${ }^{3}$ Jeanette S Torres, ${ }^{1}$ Elizabeth Linos, ${ }^{4}$ Victoria Plaut, ${ }^{5}$ \\ Christina Mangurian, ${ }^{6}$ Esther Choo, ${ }^{7}$ Eleni Linos ${ }^{1}$
}

${ }^{1}$ Program for Clinical Research,

Department of Dermatology,

University of California, San

Francisco, San Francisco,

California, USA

${ }^{2}$ Palo Alto Medical Foundation

Research Institute, Palo Alto, California, USA

${ }^{3}$ Santa Clara Valley Medical Center, San Jose, California, USA

${ }^{4}$ Goldman School of Public

Policy, University of California,

Berkeley, California, USA

${ }^{5}$ University of California,

Berkeley School of Law,

Berkeley, California, USA

${ }^{6}$ Department of Psychiatry,

University of California, San

Francisco, California, USA

${ }^{7}$ Center for Policy and Research

in Emergency Medicine,

Department of Emergency

Medicine, Oregon Health \&

Science University, Portland,

OR, USA

Correspondence to: Eleni Linos eleni.linos@ucsf.edu

Additional material is published online only. To view please visit the journal online.

Cite this as: $B M J$ 2018;363:k4867 http://dx.doi.org/10.1136/bmj.k4867

Accepted: 20 November 2018

\section{ABSTRACT}

OBJECTIVES

To report woman physicians' experiences, in their own words, of discrimination based on their role as a mother.

\section{DESIGN}

Qualitative analysis of physician mothers' free-text responses to the open question: "We want to hear your story and experience. Please share" included in questions about workplace discrimination. Three analysts iteratively formulated a structured codebook, then applied codes after inter-coder reliability scores indicated high concordance. The relationships among themes and sub-themes were organized into a conceptual model illustrated by exemplary quotes.

\section{PARTICIPANTS}

Respondents to an anonymous, voluntary online survey about the health and wellbeing of physician mothers posted on a Facebook group, the Physician Moms Group, an online community of US physicians who identify as mothers.

\section{RESULTS}

We analyzed 947 free-text responses. Participants provide diverse and vivid descriptions of experiences of maternal discrimination. Gendered job expectations, financial inequalities (including lower pay than equally qualified colleagues and more unpaid work), limited opportunities for advancement, lack of support during the pregnancy and postpartum period, and challenging work-life balance are some of the key themes identified. In addition, participants' quotes show several potential structural drivers of maternal discrimination and describe the downstream consequences of maternal discrimination

\section{WHAT IS ALREADY KNOWN ON THIS TOPIC}

Over two thirds of women physicians report experiencing gender discrimination and a third of academic women physicians report having experienced sexual harassment

Discrimination may also explain part of the gender gap in leadership roles, and gender pay discrepancy in medicine

\section{WHAT THIS STUDY ADDS}

This large qualitative study of discrimination among physician mothers reveals frequent, persistent, and sometimes blatant discrimination experienced by physician mothers based on their status as mothers

Some of the experiences of maternal discrimination are consistent with those reported by women across professions, but there are unique aspects of medical training and the medical profession that exacerbate maternal discrimination

on the physician herself, her career, family, and the healthcare system.

\section{CONCLUSIONS}

These findings provide a view of maternal discrimination directly from the perspective of those who experience it. Women physicians report a range of previously uncharacterized ways in which they experience maternal discrimination. While certain aspects of these experiences are consistent with those reported by women across other professions, there are unique aspects of medical training and the medical profession that perpetuate maternal discrimination.

\section{Introduction}

The proportion of medical school graduates who are women has increased from $7 \%$ in 1965 to $53 \%$ in $2017 .{ }^{1-3}$ However, there is ample evidence of persistent gender discrimination against women physicians, from disrespectful treatment to persistent pay discrepancies that are evident among early and mid-career academic physicians as well as those in private practice. ${ }^{4-8}$ When directly asked, $70-77 \%$ of women physicians report experiencing gender discrimination, ${ }^{9} 10$ and $30 \%$ of academic women physicians report having experienced sexual harassment. ${ }^{9}$ Discrimination may also explain at least part of the gender gap in leadership roles, a phenomenon particularly well documented in academic medicine: while women comprise $46 \%$ of US residents (doctors in training), they comprise only $38 \%$ of faculty (academic medicine) positions, $21 \%$ of full professors, and 15\% of department chairs. ${ }^{11} 12$

In addition women physicians, an estimated $80 \%$ of whom are or will become mothers, also report experiencing "maternal discrimination" based specifically on their motherhood status. ${ }^{13}$ However, there are limited data on the experience of maternal discrimination or its sequelae on the careers of women in medicine. The primary goals of this study were to (a) characterize the ways in which physician mothers experience maternal discrimination; (b) examine cultural and structural characteristics of the healthcare system that may facilitate maternal discrimination; (c) describe a range of outcomes of maternal discrimination; and $(d)$ identify organizational targets that have the potential to minimize discrimination against physician mothers.

\section{Methods}

\section{Data collection}

Details of study methods have been previously published. ${ }^{10}$ Briefly, data were collected prospectively through a voluntary, anonymous, opt-in, online survey 
with informed consent. The survey was developed by the research team on secure REDcap survey software. The link to the survey was posted on a Facebook group: the Physician Moms Group, a diverse online community of physicians who identify as mothers. At the time of the survey in June 2016, there were approximately 60000 members, of whom 34956 were active members visiting the page at least once per month. In order to participate in the survey, participants had to click on a link that directed them to a cover page with a consent form that explained the goals of the study, and that it was voluntary and anonymous. The survey was posted three times starting June 17, 2016, and the data were collected through the end of August 2016. A total of 5782 physician mothers completed the full survey, with a participation rate of $36.0 \%$ based on the number of individuals who saw the post (estimated to be 16059 unique views), or a participation rate of $16.5 \%$ based on the total number of active members (34956), which is similar to past survey participation rates in this group. ${ }^{14}$ The vast majority of participants completed the full survey (99.2\%).

The full survey included questions about demographics, physical and reproductive health, perceived discrimination, potential workplace changes, and burnout. All questions were optional. Regarding discrimination, participants were asked: "Have you ever experienced any of the following forms of discrimination at your workplace? (Please select all that apply)," followed by the open ended statement: "We want to hear your story and experience. Please share." Comments were provided by 1009 physicians and varied in length from a short phrase to multiple paragraphs.

Ethical approval for this study was obtained from the University of California, San Francisco's institutional review board.

\section{Analysis}

Participant comments were analyzed using Dedoose, an online program for analyzing qualitative and mixed methods data (www.dedoose.com). A team of three trained qualitative researchers (MCH, ASR, JST) drew on the techniques of grounded theory in analyzing the data. This approach is designed to identify emergent social and structural patterns in human experience and to generate a theory or theories to explain these patterns. ${ }^{1516}$ First, the team read the data in their entirety and developed a structured codebook that included inductively derived codes that represented common and/or unique ideas that emerged from the data (such as missed opportunities, job changes) as well as deductively selected categories to organize the dataset. The latter included codes to identify any comments that were unrelated to either gender or maternal discrimination $(n=62)$ for exclusion, leaving 947 excerpts in the final analysis.

The analytic team conducted multiple rounds of codebook revision and interrater reliability testing until a minimum pooled kappa value of greater than 0.8 was achieved by all coders to ensure the clarity of coding definitions-including which portions of a comment related directly to maternal discriminationand their consistent application. The analytic team then reviewed and consolidated these memos into a list of key themes and subthemes designed to characterize the range of experiences of maternal discrimination as well as the processes that led to and resulted from these experiences. Only ideas relevant to maternal based (not only gender based) discrimination were included in the conceptual model. We include instances of discrimination that are tightly related to gender stereotypes (for example, that women should be warm and nurturing), which are based on traditional social roles related to motherhood. We used an iterative process to refine and organize these themes into a conceptual model. ${ }^{16}$

To evaluate the representativeness of our sample, we calculated median age, race and ethnicity, marital status, number of children, geographic location, practice type, and medical specialty of our study group and compared these demographics with women physicians reported by the 2016 Association of American Medical Colleges (AAMC) survey. ${ }^{17}$

\section{Results}

Demographic data for the full sample have been reported previously. ${ }^{10}$ The median age was 39 years (range 24-62), median number of children was two (range 0-6, where 0 children included mothers who were currently pregnant or had lost children). Overall, respondents were similar to the general population of US physicians: 74.2\% White (US 75\%), 12.9\% Asian (US 12.8\%), 8.4\% Hispanic or Latina (US 5.5\%), and $5.3 \%$ Black (US 6.3\%). Those who responded to the discrimination prompt were similar to nonrespondents in terms of demographics (age, race/ ethnicity, geographic location), and career factors (training stage, medical specialty, years in practice). Most participants were residency-trained practicing physicians (90\%) and most worked $>40$ hours per week (43\% worked 41-60 hours per week, 17\% worked $>61$ hours per week).

The themes and relationships from the 947 participant responses summarized in the conceptual model (fig 1) are organized into five broad categories related to maternal discrimination: experiences, drivers, interpersonal mediators, impacts, and modifying factors (tables 1-5).

\section{Experiences of maternal discrimination}

This category captured the diverse ways in which physician mothers experience discrimination due to pregnancy, maternity leave, breast feeding, and subsequent parental responsibilities (table 1). Many of these reported experiences of discrimination are consistent with research documenting the application of different standards for pregnant women, and the implications of these perceptions for hiring and promotion-related evaluation. ${ }^{18-21}$

Gendered performance expectations-The first theme described higher standards applied to physician 


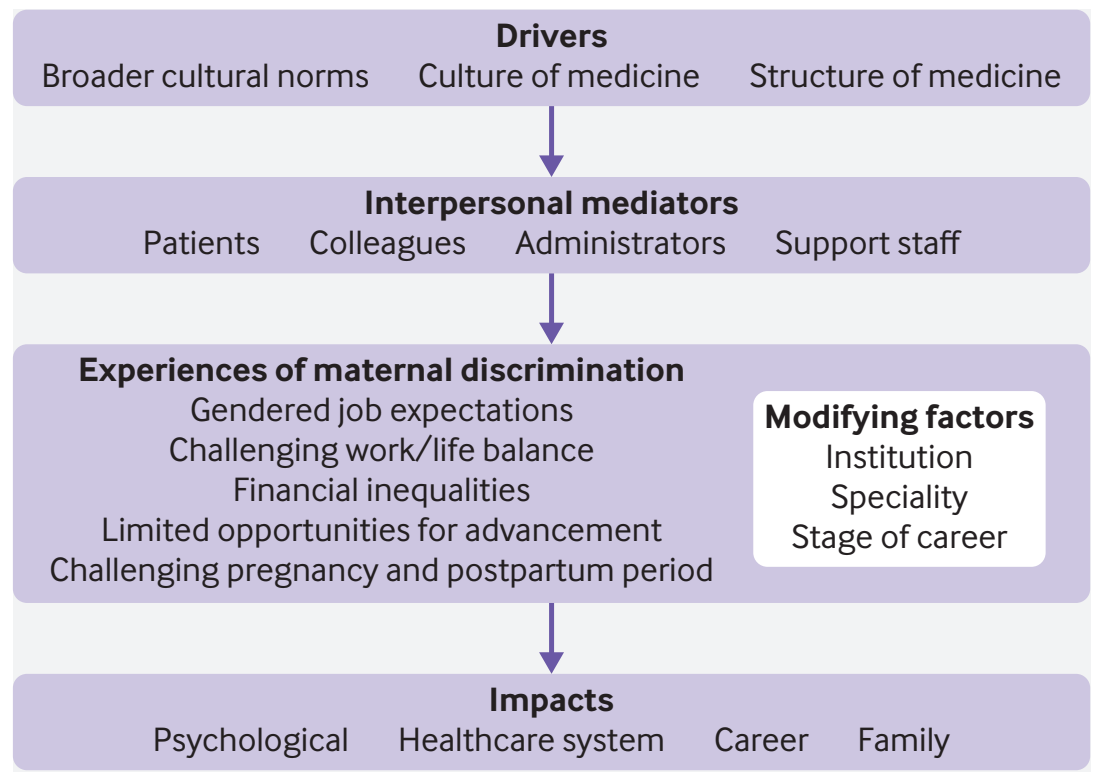

Fig 1 | Conceptual model of themes and relationships derived from physician mothers' reported experiences of discrimination

mothers than their colleagues, which physician mothers described as a requirement to prove their commitment and competence as a physician. Conversely, other participants described being held to a lower standard, in that their colleagues assumed that they were not interested or able to take on new tasks or leadership roles due to their maternal responsibilities, and, therefore, they were never offered.

Limited opportunities for advancement-The second theme included participants' descriptions of being excluded from administrative decision making because of pregnancy or after returning from maternity leave. Participants also described being passed over for leadership roles in favor of colleagues perceived as less qualified or having their contracts grossly modified or terminated in response to announcing a pregnancy or when returning from maternity leave.

Financial inequalities-This was based on participants' experiences of receiving lower salaries than colleagues with comparable (or significantly less) experience and productivity. In addition, women reported being asked or required to take on more work (such as serve on committees) without additional pay, while male colleagues were more typically compensated for additional work. This also was an issue following maternity leave, after which women reported being required to "make up" the time without pay or benefits, despite the fact that their leave time was already unpaid.

Lack of support during pregnancy and the postpartum period-This theme captured participants' descriptions of the disparagement of maternity leave and motherhood in their workplaces, including colleagues referring to maternity leave as "vacation," being pressured to forgo leave to which they were entitled, and/or being subjected to rules and expectations regarding leave that were not applied to male colleagues. Participants described feeling "punished” for their pregnancy, maternity leave, and/ or pumping breast milk through personal mistreatment or unreasonable expectations.

Challenging work-life balance-This incorporated comments about challenges finding childcare for unusual or extreme work hours, the lack of alternative work schedules to support work-life balance, and balancing home responsibilities with growing demands on physicians to complete administrative tasks after clinic hours.

\section{Drivers of maternal discrimination}

This category was developed to capture the underlying causes of maternal discrimination as perceived by participants in three themes (table 2).

Broader cultural norms included participants' descriptions of how their experiences working in medicine reflected gender norms in society, such as the expectations of women's interpersonal interactions and social, financial, family, and workplace roles, consistent with well documented gender norms and expectations in feminist sociology, anthropology, and social psychology. ${ }^{22-25}$

Culture of medicine aggregated participants' descriptions of the norms, values, and expectations underlying individuals' social roles and interpersonal interactions specifically within the medical workplace. The culture of medicine was evidenced by several assumptions or expectations: that mothers cannot be successful doctors, that doctors cannot be good mothers, that women physicians should delay childbearing, that childbearing necessarily ruins women physicians' careers, and that physician mothers do not value their careers.

Structure of medicine was based on participants' perceptions of ways in which the administrative, procedural, and physical structures of the medical workplace perpetuated maternal discrimination, including policies and procedures that limit maternity leave, the lack of coverage and flexibility in physician schedules, the lack of physical space and time to breastfeed or pump milk, the long (and often overnight) work hours generally required of physicians, and incentive structures that financially penalize mothers for taking maternity leave or pumping breaks.

Interpersonal mediators of maternal discrimination This category captures the avenues by which the drivers of discrimination manifest in interactions in the workplace(table 3). This is consistent withlongstanding sociological theory that interpersonal interactions mediate the relationship between individual experience and culture norms and expectations of gender, and with more recent research on the role of implicit bias in perpetuating discrimination. ${ }^{4}$ This category is organized by the core groups that physician mothers interact with in the workplace, including physician colleagues, administrators, support staff (such as nurses), and patients and their families. These examples demonstrate the personal and sometimes 


\section{Table 1 | Experiences of maternal discrimination as described by self identifying physician mothers among 947 qualitative excerpts, by theme and subtheme}

Theme and subtheme
Gendered job expectations
Higher standards for physician mothers

Lower expectations or pre-

sumed disinterest

\section{Exemplary quotes}

"Was held to a higher performance than my peers when pregnant"-Participant 426

“Despite meeting my RVU goals (even with 10 weeks of maternity leave), I was told I did not qualify for a raise because I hadn't been productive enough"-Participant 234

“Unconscious bias is prevalent. 'You don't want to deal with that,-you have small kids'”-Participant 459

"My impression is that I'm not invited to some 'extra'-type work things because it's assumed that l'll opt out because I have young children. Basically, someone else is opting out for me"-Participant 978

“I was not considered for the assistant medical director position because it was felt it would 'take away from my time at home' by my director. I am part time right now, but would have considered increasing my hours for this new opportunity. Instead, the position was given without anyone's input to a fresh out of residency male graduate with years less of clinical and administrative experience"-Participant 671

\section{Limited opportunities for advancement}

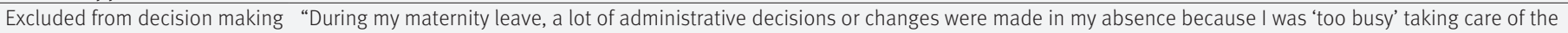
new baby. I would have still appreciated being included in the workplace decisions and changes that were made"-Participant 554 "Frequently not included in projects and/or decisions after I announced I was pregnant. Again not included in decisions after I returned from maternity leave"-Participant 202

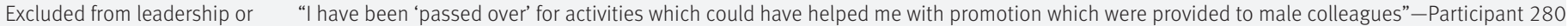
career advancement

"[l] didn't get chief resident though selected by majority due to pregnancy"-Participant 6

"Most times when a leadership opportunity presents I volunteer. For many years, all I hear is that a male peer has more experience or needs to find his 'niche' or that I need to spend more time with my family"-Participant 738

"Although I was the senior neurointensivist in our hospital, my colleague (male, fresh out of fellowship) was given the directorship position when the previous director left"-Participant 516

Job or contract changes or

termination said while I was gone, they are no [longer] having part time and I would need to work full time. After talking with some lawyers it seemed that even though they were breaking the contract and going against FMLA, it would be a difficult course. I left and got another job after being there for almost 9 years"-Participant 402

"I was told the practice would lose money because of my pregnancy and mat[ernity] leave. I was told my ankles would swell, my baby would end up in the NICU, and then I would want to be there with her so the practice would lose even MORE money and because of all this I need to accept a new contract with lower pay, no partner track, and no right of first refusal if the practice was ever sold. This is just a small amount of what happened and what was said to me. I signed the new contract because I was threatened I would lose my job and believed I really had no choice"-Participant 292

"I was given an ultimatum when out on maternity leave to return under a contract that was different than when I left or not return at all. I was a part-time salaried physician and forced to return full time"-Participant 406

"I was fired 2 weeks after having a baby without any warning whatsoever. Not only that, they did it over the phone, then had all my hospital privileges revoked overnight since I no longer had a physical office"-Participant 276

\section{Financial inequalities}

Lower pay than equal or less qualified colleagues

"I was given a smaller raise than my male colleague at the same level despite bringing in more revenue. When I asked for a raise my Chair told me that my husband should get a job. When I got back from maternity leave, he had given one of my OR days to another colleague"-Participant 666

"I definitely have been paid less than male counterparts. I even discovered that recent graduates I had trained were making more money than I did (10 years more experience)"-Participant 205

More work for no more pay "There exist some hospital committees that have paid stipends for membership. These are split between the male partners- not one male partner is unpaid for his administrative work...have asked the group multiple times to just strike stipends and pay a set hourly rate for any and all administrative tasks.... met with a great deal of push-back"-Participant 43

"As a resident, our GME office decided that residents taking leave (would only apply to maternity leave essentially) would have to make up the time without benefits or pay"-Participant 16

"When I was working as a hospitalist (which I am not anymore because of burnout), there were 4 in our group who worked part time. We were all women and all mothers. The group's director (who was a single man without children) wanted to require us to take the same amount of holiday call as our full time counterparts. He also would not allow us to take our CME day (to which we were contractually obligated) because he felt that working part time should allow us plenty of time to do CME on our own time"-Participant 1007

\section{Lack of support during pregnancy or postpartum period}

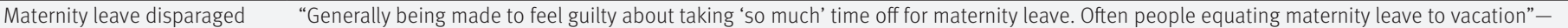
Participant 170

"My son was born premature and was in the NICU when I was an intern. The upper level resident I was on a rotation with asked me how I was 'enjoying my vacation' in reference to my maternity leave. I spent that maternity leave in the NICU. It wasn't a vacation. And I finished residency 2 months later than everyone else, as well as using all of my vacation/sick leave"-Participant 111

"After coming back from a mere 6 weeks of maternity leave my boss said, 'Must have been nice to have had all that time off. I've never had that much time off!'”-Participant 9

“Told that taking maternity leave was a bother to everyone and that I can't just "keep going on vacation for 6 weeks"”-Participant 30

"Punishment" for maternity “Unpaid maternity leave AND obligation to makeup missed calls. However, partners who take medical leave are not required to repay call”leave Participant 150

"The office manager informed me that my partners wanted to charge me more overhead cost while I was on maternity leave. Meanwhile a male partner took all of July off every year for over 20 years"-Participant 369

"I took the same time off afforded to any resident in my program ( 3 weeks vacation +1 month reading elective), yet somehow was made to suffer considerably since I was on 'maternity leave'”-Participant 164

"My male coworker and I both work as 0.8 FTEs but he was able to keep full-time status and benefits while I was suddenly changed to part-time after returning from maternity leave. We work in the same practice. When I brought this up with my department administration, I was told that I could just add myself to my husband's benefits package"-Participant 321

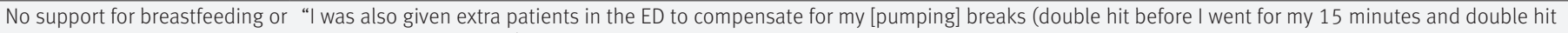
pumping again when I came back) even though I could see on the tracking board that the other team didn't get any patients"-Participant 66 "As a resident pumping for my 3 month old child (after coming back to work 4 weeks postpartum) I was told by my associate program director that my 'personal life was interfering with my ability to do perform my work responsibilities' because of taking breaks to pump every 4-6 hours. Despite the fact that I was pumping in a public work space surrounded by attendings, residents, medical students, and occasionally consultants from other services so as not to miss any important clinical work or decisions rather than pump in private"-Participant 166 


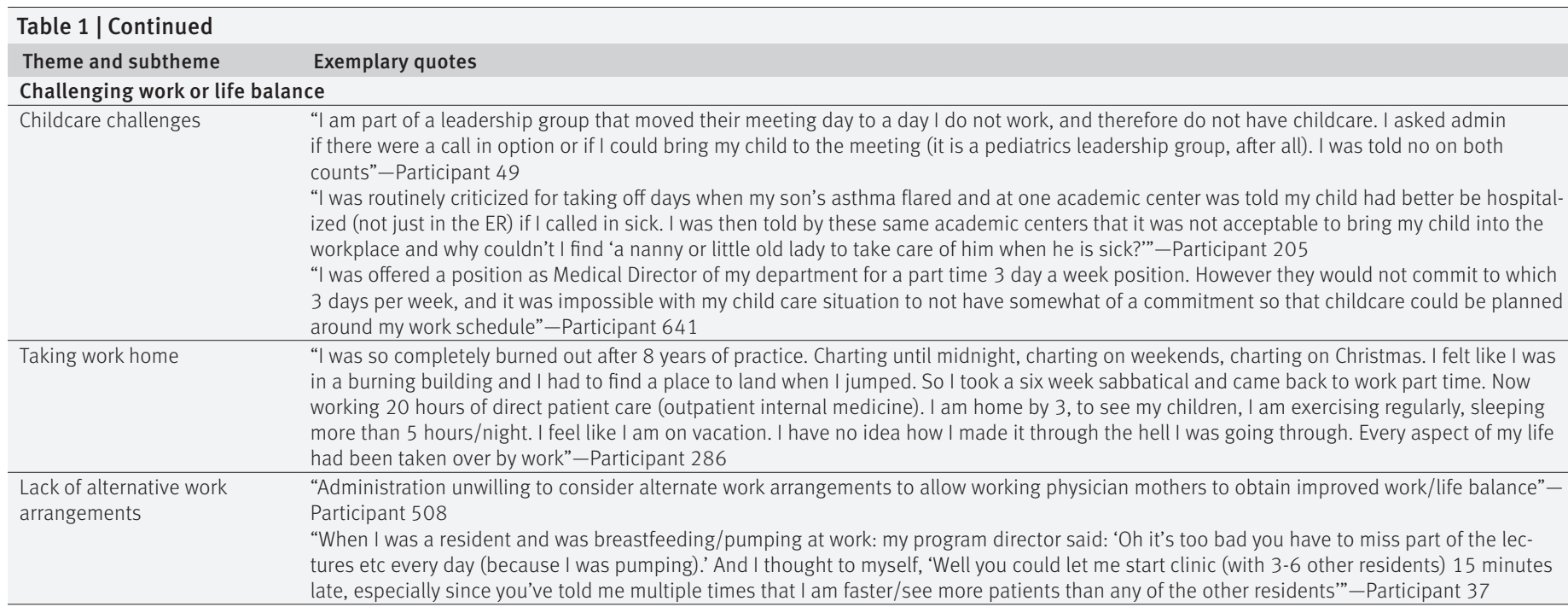

blatant ways in which discrimination is experienced by physician mothers.

\section{Impacts of maternal discrimination}

This category includes themes focused on the downstream effects of maternal discrimination on the women, their families, and the healthcare system (table 4).

Psychological, captures participants' descriptions of the psychological consequences of maternal discrimination, including extreme stress due to work and family demands, guilt from feeling unable to meet those demands, and fear of reporting discrimination due to possible retaliation.

Career focuses on the negative impacts of maternal discrimination on careers. A common example cited by participants was switching from full time to part time work, a "choice" that was not always described as voluntary. Participants also described leaving academic or clinical medicine, leaving group practice for solo private practice, or leaving medicine altogether in response to an unfriendly work environment characterized by maternal discrimination. Many women's career changes also came with immediate financial consequences, including lower salaries and fewer benefits. Additional financial consequences included being denied earned salary increases or bonuses due to maternity leave (despite reaching or exceeding productivity goals), being denied higher paid positions due to motherhood, paying full time overhead costs despite holding a part time position, and forgoing pay to recover from birth or take pumping breaks.

Family outlines the impact of maternal discrimination on the families and children of physician mothers. The negative financial consequences described above also undermined participants' family's financial stability, particularly for mothers who were the primary breadwinner in their family. In addition, women were expected to perform tasks while pregnant that presented risks to the fetus (such as exposure to communicable diseases known to cause fetal defects, continuing work while in preterm labor or while having a miscarriage) and many described prematurely curtailing breastfeeding or pumping due to lack of time, space, and support. Participants also described generally feeling that they were expected to ignore the advice regarding child care that they were giving to their own patients due to inflexibility in their workplace.

Healthcare system-Under this theme, participants described negative impacts on the healthcare system and their patients due to maternal and gender discrimination. This included potential impacts on healthcare quality due to their orders being ignored (whereas those of male colleagues were not), putting patient safety at risk. In addition, participants described how their part time status, which many found to be the only option for work-life balance, meant they were automatically excluded from leadership and other opportunities to be involved in decision making. Participants also described being passed over for these leadership positions in favor of less qualified colleagues.

\section{Modifying factors}

Finally, table 5 captures the modifying factors that participants' pointed to as either perpetuating or mitigating maternal discrimination.

\section{Discussion}

Our findings suggest insidious, persistent, and sometimes blatant discrimination experienced by physicians, based on their status as mothers. While these experiences may be mitigated or exacerbated by stage of training, institution, and choice of specialty, participants' reports suggest these experiences are pervasive. Our conceptual model illustrates the role of broader cultural norms and expectations of mothers, as well as both the culture and structure of the medical workplace in perpetuating maternal discrimination. In addition, our data suggest multiple ways in which maternal discrimination has short and long term consequences, not only on the individual 


\section{Table 2 | Drivers of maternal discrimination as described by self identifying physician mothers among 947 qualitative excerpts, by theme and subtheme}

Theme and subtheme Exemplary quotes

\section{Broader cultural norms}

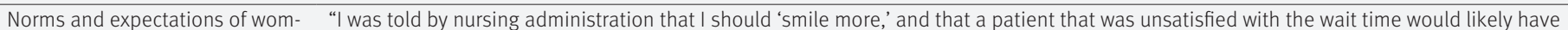
en's interpersonal interactions

not complained if I just 'smiled more"”-Participant 3

"When I ask politely for things to be done a certain way, the support staff says, 'Oh, it's her way, or the highway,' or 'She's very particular,' or 'She's difficult to work with.' When a male colleague had a screaming fit, including swearing, in the hallway because a patient was added into his schedule at the last minute, the support staff told this as a humorous anecdote"-Participant 37

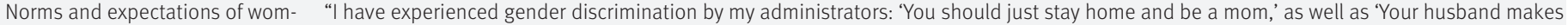
en's financial or family role enough money; why do you work?'”-Participant 64

"My first chair and an academic institution gave the man I hired, in the lower academic rank, who I supervised, the title of director of clinical services. My boss explained that I would still do the job but the title needed to go to him so that he could give him a raise because his wife just had her second baby and wanted to stay home and that was a lifestyle my boss wanted to promote"-Participant 4

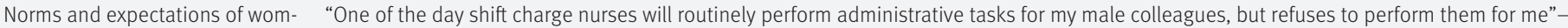
en's workplace role Participant 3

“Just today my attending made the comment (to the male coresident and med student sitting at the desk next to me in a small room) 'I don't take orders well, unless it's 'sit down while I make you dinner' ...Gentlemen, 30 years ago when you said jump, a woman said how high.' It was very uncomfortable. This same attending left negative feedback for me on my last rotation - I definitely feel there's gender bias" Participant 99

\section{Culture of medicine}

Assumption that mothers cannot "Several times in the OR I have been told in one way or another I will not amount to much because I was a female and having a family"be successful doctors

\section{Participant 121}

"While applying to medical school I was newly married, I was consistently asked on interviews when I was planning on having a baby. When my husband and I were applying to residency at the same time he was lauded for having a family, I was always asked if I thought I could handle residency and being a wife and mother"-Participant 894

"As a pregnant 3rd year med student, a male attending on my first rotation told me I had to decide if I was going to be a good mother or a good doctor, that I couldn't be both"-Participant 13

Expectation that women should “Other female physicians bragged about their sticking to managements 'not getting pregnant' implied requests"-Participant 123 delay or avoid childbearing "The attendings who ran my residency program were resentful that I got pregnant during residency"-Participant 192

Perception that childbearing ruins "I was subjected to near daily comments about how I ruined a career in academics because I got pregnant in residency"-Participant 14 women's careers

"I have been told not to have any more children and that by having a child I would have to 'start over' with my practice"-Participant 62 "The woman before me who got pregnant during residency was told by the chairman that 'she'd just ruined her career" - Participant 192

Perception that mothers aren't committed to their careers “In residency, being told that residents who have babies 'obviously don't want to work”- - Participant 182

"There was an instance where all the female physicians were told they weren't working their contractual hours. So I actually spent the time going through each physician's schedule and demonstrated that the male physicians had the same leeway time (contract states 40 hours but you actually are scheduled 36 hours, etc) and I worked actually the 2 nd most number of hours out of 11 physicians only trailing the lead by an hour. They then shelved their complaints but I was ticked off that only the 3 female physicians who had children were targeted"Participant 138

"There was a culture among other residents to complain about female attendings. Although these attendings were contracted for part time, they were often referred to as 'lazy' because they desired spending time with their children"-Participant 114

Structure of medicine

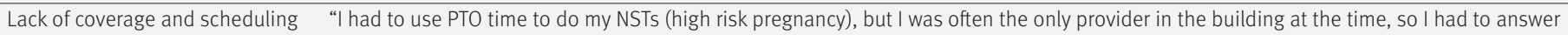
flexibility messages and deal with emergencies during this time"-Participant 61

“I asked for a schedule accommodation due to monitoring for my gestational diabetes and was met with 'ain’t no way' until my OB/GYN threatened to simply certify disability. I didn't even ask for accommodations for pumping”-Participant 52

Lack of space and time for breast- "No ED lactation room. Requested numerous times. Told to use an admin nurses office, but she (rightfully) got tired of being kicked out of feeding or pumping her own office for everyone to pump...Plus no sink in the office. RN locker room only has one toilet and no privacy anywhere. We usually sneak off to a call room but it's infested with cockroaches. For years. Complained but nobody cares"-Participant 24

"Had to pump in the bathroom while breastfeeding because it took longer than my 15 min break to get out of the OR, to the one and only pumping room in our huge hospital, set up, pump, and back to OR”-Participant 16

Lack of or limited maternity leave “Told 'pregnancy was a choice...not an illness' so shouldn’t be allowed to use sick leave for maternity leave”-Participant 145

policies "I saved my vacation time up to use for maternity leave however the hospital said that I could not use this as vacation and my vacation expired a month later. So I didn't get paid my vacation and it expired before I could get back from maternity leave. Sadly, I only took 4 weeks off because that's all I could barely afford while not being paid"-Participant 273

"Lost my benefits of paid maternity leave with my second child without being notified by my employer so nearly went bankrupt to stay home with my 2 nd for 8 weeks of FMLA leave in addition to being forced to use all my vacation time"-Participant 18

Long or night work hours required "I was put on call every other night my last 2 months of pregnancy and my first 2 months back after maternity leave, which I managed by using all my sick leave, vacation days, and short term disability"-Participant 192

"Felt overworked pregnant as in scheduled brutal night shifts and worked up until night before due date"-Participant 24

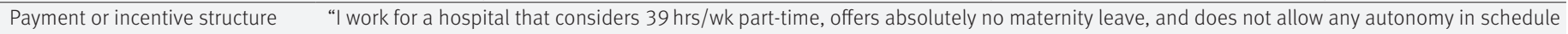
inflexible to motherhood making....but only for the female physicians"-Participant 94

"I have no maternity benefits. I had to make up my call, which means I will be q4 for 16 months to take off 3 weeks of call before delivery and 8 after. During this time, I continued to have to pay overhead"-Participant 175

physician, but also on her family and the larger healthcare system.

These findings extend research on gender discrimination in the medical workplace by focusing on motherhood and, notably, provide a view of maternal discrimination directly from the perspective of those who experience it. These reported experiences are consistent with a wide range of sociological and psychological studies documenting the stereotyping of mothers and the implications for workplace outcomes. $^{2025}$ These include gendered expectations of women's interpersonal interactions and gendered assumptions about workplace ambition and professional capabilities.

Our findings also contribute to a broader debate on the causal explanations of the "motherhood penalty"- 


\begin{tabular}{|c|c|}
\hline Group & Exemplary quotes \\
\hline Administration or leadership & $\begin{array}{l}\text { "Chief of service once told me that he did not want to hire more women because of people being out for maternity leave and calling in sick with } \\
\text { kids"-Participant } 538 \\
\text { "I was told that my hospital 'legally had to offer me } 12 \text { weeks of unpaid FMLA but I was told not to take that long because they couldn't afford } \\
\text { locums that long"-Participant } 126\end{array}$ \\
\hline Colleagues & $\begin{array}{l}\text { "Many (male) colleagues openly complain about too many women in our group, too many pregnant or could get pregnant women"-Participant } \\
24 \\
\text { "Was reminded every day by my peers on how 'irresponsible' I was getting pregnant"-Participant } 426 \\
\text { "Attendings harassed me for pumping more than once during my shifts (12 hour shifts that usually were } 13 \text { or } 14 \text { hours five days a week)"- } \\
\text { Participant } 14\end{array}$ \\
\hline Support staff & $\begin{array}{l}\text { "My office manager gave me a very hard time about scheduling breastfeeding, especially when a meeting was scheduled during my pumping } \\
\text { time and she did not want to reschedule my patients to give me time to pump”-Participant } 125 \\
\text { "Consistently disrespected by nursing staff, respiratory therapists, case managers, etc. Last call, orders I placed did not get done because, the RT } \\
\text { 'didn’t think they were indicated,' and called me 'doctor' using actual air quotes....This never happens to my male colleagues"-Participant } 544\end{array}$ \\
\hline Patients & $\begin{array}{l}\text { "I am the only female emergency medicine physician at my location - yet the only discrimination I feel is from patients. My peers don't under- } \\
\text { stand the constant 'When will I see the doctor?' comments"-Participant } 51 \\
\text { "Patients and their families sometimes are condescending, ignorant. (They assume I'm not a physician because I'm female)"-Participant } 723\end{array}$ \\
\hline
\end{tabular}

the empirical finding that mothers face an additional wage penalty (over and above any gender wage penalty) even as men enjoy a "fatherhood bonus." ${ }^{2627}$ Although prior research has shown that the motherhood penalty is a problem among women from low socioeconomic status, our data suggest that this problem also applies to women in higher paying fields such as medicine. ${ }^{28}$

Other aspects of physician mothers' experiences are specific to the environment of healthcare and institutional policies and norms around physician training, suggesting ways in which maternal discrimination may manifest for physicians in particular. The intensive and extended time course of medical training provides a particular conundrum for women physicians, as their training is rarely complete until they are already nearing the end of their childbearing years.

Some evidence suggests that gender-specific "work preferences" and "life values" may explain some of the gender differences found among parents in the workplace. ${ }^{29}$ Indeed, when looking at national data, many speculate that women choosing "motherhoodfriendly" occupations may account for the persistent wage gap, and find a much stronger motherhood penalty among low income and low wage workers. ${ }^{28}$ These data underscore that "work preferences" and "life values" may themselves be shaped by the limitations of the specific work environment mothers face, including physician mothers, and what seems to be a career "choice" may actually stem from the internalized preclusion of other options and interests. ${ }^{19} 2930$ In fact, exposure to gender discrimination and sexual harassment may influence medical students' choice of specialty. ${ }^{31}$ Unfortunately, when motherhood itself is viewed as a choice, mothers' incomes are penalized more. $^{32}$

Certain aspects of the structure of medicine and medical training are unique, deeply ingrained, and may inadvertently discriminate against women who become mothers. The limitations of this work environment perpetuates a cycle in which women, and mothers in particular, are excluded from leadership positions in which they might otherwise be able to drive incremental change by advocating for more family- friendly policies in their institutions. Identification of specific structural biases that contribute to maternal discrimination-such as institutional policies that exclude part time physicians from leadership rolesis essential to identifying avenues for intervention. In addition, our findings indicate that maternal discrimination varies by institution, suggesting that solutions exist. Further research examining "positive deviants"-that is, institutions where physician mothers report better work-life balance and supportcould provide models for combating discrimination elsewhere. Further, we see men as allies who can help make long lasting change by advocating for familyfriendly policies.

It is noteworthy that some of the downstream effects of maternal discrimination might be mitigated by policies and procedures: flexible schedules including shift work, on-site high quality child care, longer paid parental leave, etc. A recent analysis of parental leave policies of top US medical schools showed high variability in the duration of paid leave, and that duration of leave was often negotiable and at the discretion of supervisors. ${ }^{33}$

However, there is promising evidence that efforts to increase awareness of family accommodation policies (including presentations, workshops, and web or social media presence) improves uptake of those policies. ${ }^{34}$ The pervasive misconceptions and negative cultural norms around maternity leave documented in this study strengthen the argument that decisions about maternity leave cannot be left to the discretion of individual employers if they are to be implemented consistently. Because generous family-friendly policies may unintentionally exacerbate discrimination against women in the workplace, research is needed to understand how to most effectively create structural changes to the work environment given the underlying cultural biases against mothers. ${ }^{35} 36$

Although our participants were mostly from the United States, the problems highlighted are not unique to the US: a cross national comparison of 22 countries found that wage penalties associated with motherhood were smaller in countries with public policies that maintain mothers' labor market attachment (for 


\section{Table 4 | Impacts of maternal discrimination as described by self identifying physician mothers among 947 qualitative excerpts, by theme and subtheme}

Theme and subtheme Exemplary quotes

Psychological

Stress

"I was very depressed after my first child because of the stress of going to work so quickly and trying to balance all demands of being a mom, work and fellowship"-Participant 190

"I was never suicidal, but I can understand why many physicians feel that suicide is the only way out of the unrelenting demands (many self-imposed) of medicine"-Participant 286

Guilt "I have had another female doctor who works part time make me feel guilty because I work full time and have "someone else raise my kids"'-Participant 65

"I am tired of feeling like I am a bad mom when I am being a great doctor...and like a bad doctor when I am putting my children first"Participant 708

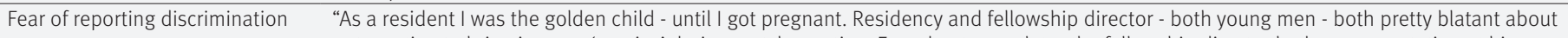
not wanting to bring in more 'ovaries' during match meeting. Found out years later the fellowship director had same reputation at his previous institution. There are no repercussions for these guys (they are still there 7 years later). I've never said anything - I'm afraid of being blackballed in a small specialty"-Participant 210

"When my boss retired four years later, I found out from my new boss that indeed this younger [male] physician was making $30 \%$ more than me, with the title of the job he did not do, I did... I should have gone to the dean when the situation first emerged years ago, and then I should've gone to the dean even after I had the new boss. However I didn't understand my options and I was terrified of making trouble"Participant 4

\section{Career}

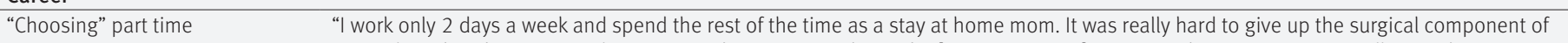
gynecology, but the patient volume required to warrant a day in the [operating room] was something I just was not willing to do. We're a 2 physician family and I am fortunate that my spouse's income can support this decision"-Participant 745

"My employer attempted to deny me FMLA for baby bonding time, which was blatantly illegal and downright insulting given my hard work and commitment to the group. Ultimately, I was so upset by this that I quit on the spot, currently transitioning to a new job that provides me more support and fewer hours so that I can be a more present mother and wife"-Participant 106

"I worked 0.8 FTE when my kids were small. I was unable to vote on peer promotion and was overlooked for several initiatives due to my 'part time' status despite roughly a 40 hour workweek"-Participant 216

"I did have burnout resulting in a small breakdown 3 years ago. I was working insane hours with 2 young children. I spent 3 days in bed and through the amazing support of my husband I healed. We decided that I would work part time after that"-Participant 450

Other career changes "I have been feeling burnt out for over 1 year after only being in practice for 2 years. Although I love teaching and research, I recently accepted a private practice position where my clinical workload will be about $2 / 3$ and no teaching/research responsibilities. My salary will be slightly higher and l'll get a $\$ 50 \mathrm{k}$ retirement contribution/year...Honestly, I think the politics and misogyny are worse in academics than private practice"-Participant 93

"I'm now opening my own practice but that has its own pitfalls as a mother. But at least I have control and no more snide remarks from management to deal with"-Participant 138

"As a busy Ob-Gyn with 3 kids and a husband who travels every week, I have struggled with juggling work and family. My partners were shocked when I told them I was going to retire at age 45. I gave them a year's notice and helped interview new doctors to replace me"Participant 518

"Even though I receive excellent evaluations, graduated in the top $10 \%$ of my medical school class, and am at an amazing residency program, the environment and continuous disrespect from non-physicians and families makes me want to leave medicine sometimes"Participant 544

Financial penalties $\quad$ "Because I work part time, I cannot be considered for partnership, benefits, etc. my salary was much less, less vacation time, no CME. No option for loan repayment because I'm part time. But I was required to take the same amount of call"-Participant 22

"Only the full-time salaried employees got the raises, but all of the part-time employees (who are supposed to make a corresponding fraction of the full-time salary) were denied. At my institution, the vast majority of part-time physicians in my department are women who have child care and family care obligations"-Participant 940

\section{Family}

Risks to pregnancy

"As a trainee in fellowship, despite having preterm contractions and cervical changes, my clinic kept going in order to ge [sic] any time

after"-Participant 17

“Had a miscarriage (bleeding through my clothes) in the OR and my senior resident wouldn't find someone to relieve me because he 'didn't believe I was really sick"'-Participant 16

No sympathy when a meningitis patient or shingles patient needed to be seen. I just gowned and masked and risked my babies [sic] health. Participant 24

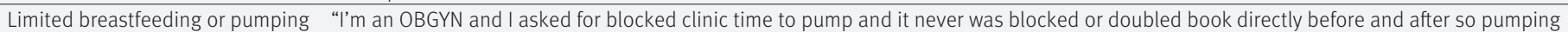
was a nightmare. It's no surprise I only made it 6 months with pumping"-Participant 273

"Also when pumping no time is allowed; specifically, you are expected to do just as much with less time. l've now stopped pumping"Participant 466

Can't follow doctor's orders "As a pediatrician, I routinely felt we gave mothers in our academic clinics advice that we as pediatrician mothers were not allowed to
follow"-Participant 205

\section{Healthcare system}

Impact on healthcare quality

"I have been ignored in a code situation where I told a nurse to bag a patient slower because he was obstructing, and I have never been
apologized to in any of these scenarios"-Participant 544

"I called an emergency [caesarean section] and the nurses didn't bring the patient into the OR even though heart tones were in the 40 s and the baby was growth restricted so I couldn't do an operative delivery if it was necessary"-Participant 55

"I resigned from that position after nearly four years due to significant burnout. I was unwilling to continue to compromise my patients' safety"-Participant 281

"I wanted to transfer an OB patient out to deliver in a hospital that had a NICU because it was a preterm delivery and we had policy to not deliver at less than 36 weeks. The OB was a male who did not transfer and didn't even discuss the situation. He talked down to me and delivered the patient"-Participant 296

Impact on healthcare leadership "Men given leadership positions regardless of their managerial skills and despite the fact that women outnumber men in our group"Participant 231

"I was a medical director of an UC, there were 5 of us in total, the 2 male directors were held in higher regard. One left as wife got a fellowship, a second was offered a promotion while three females were not. Only to find out the male who was offered a promotion was stealing from the company"-Participant 74 


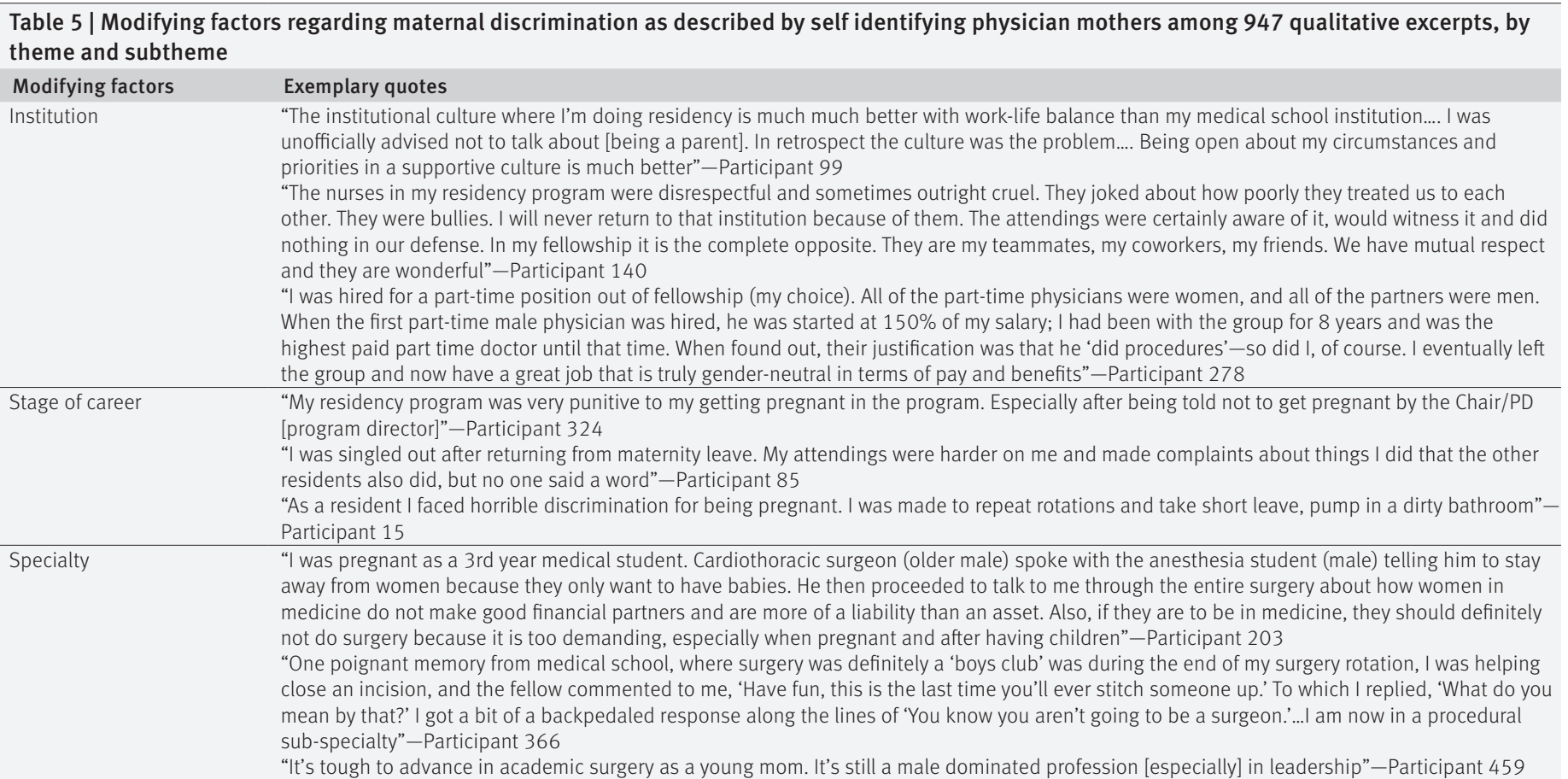

example, moderate length job-protected leaves, public funded childcare, tax rate policies that tax secondearners at a lower rate, and paternity leave). The highest motherhood penalties were found in countries with short leave policies, but the penalty was also high in countries with long leave policies (perhaps because they reinforce maternal stereotypes and weaken labor force attachment). ${ }^{37}$

\section{Strengths and limitations}

This study is the largest qualitative study to examine physicians' experiences of maternal discrimination to date, and one of the largest qualitative studies on maternal discrimination in any profession, providing an extremely rich dataset. However, the findings of this exploratory analysis should be viewed as preliminary given the limitations of the methodology. The dataset is limited to mothers, and we therefore did not have the opportunity to study women physicians who are not mothers, or fathers, and penalties they face. Entirely disentangling the effects of gender versus motherhood on discrimination was not possible with these data. We also did not have the perspective of employers or cultural context for these motivations. For instance, administrators may have to make difficult choices regarding individual employees based on broader organizational constraints. Additionally, the relatively low participation rate could bias responses, and we recognize that this is highly selected voluntary sample of participants. Women who experienced discrimination may have been more likely to respond, overestimating prevalence of discrimination. Conversely, the prevalence of discrimination could have been underestimated if women who left the profession due to the negative impacts of discrimination were not included in our sample of mostly practicing physicians.
We believe response bias is likely not a major concern because our survey was not limited to questions about discrimination, and was a broader survey about health and wellbeing of participants. In addition, research suggests few differences between responders and non-responders in physician surveys. ${ }^{38}$ Another limitation is that the relative lack of racial diversity in the sample did not permit us to examine how maternal discrimination and racial discrimination may intersect.

\section{Conclusion and next steps}

These preliminary findings suggest insidious, persistent, and sometimes blatant manifestations of discrimination experienced by physician mothers. The cyclical nature of maternal discrimination in medicine is concerning because it reinforces and perpetuates key drivers of maternal discrimination in medical training and practice. As we strive to build more equitable workplaces, our findings suggest that challenging norms around motherhood in the medical workplace, as well as structural changes that address pregnancy, parental leave, and childcare, are needed in order to mitigate the impacts of maternal discrimination in medicine.

$\mathrm{MCH}$ and ASR contributed equally to this work.

We thank Lily Morrison for technical support with this manuscript, and Hala Sabry and Dina Seif for their help with recruitment.

Contributors: MCH and Eleni L conceived of the study and analysis plan. MCH, JST, and ASR analyzed the data. MCH drafted the conceptual model. MCH and ASR wrote the first draft of the manuscript. All authors contributed to interpretation of the data, edited the manuscript, and approved of the final manuscript. Eleni $\mathrm{L}$ had full access to all the data in the study and had final responsibility for the decision to submit for publication.

Funding: Eleni L is supported by the National Cancer Institute (grant No R21CA212201, the National Institute of Aging (grant K76AG054631), and the National Institute of Health (grant DP2CA225433); VP is supported by NSF \#1535435; and CM 
is supported by grants $\mathrm{K} 23 \mathrm{MH} 093689$, R01MH112420, and R03DK101857.

Competing interests: All authors have completed the ICMJE uniform disclosure form at www.icmje.org/coi_disclosure.pdf and declare: no support from any organization for the submitted work.

Ethical approval: Obtained from University of California, San Francisco's institutional review board.

Data sharing: No additional data are available.

The lead author affirms that this manuscript is an honest, accurate, and transparent account of the study being reported; that no important aspects of the study have been omitted; and that any discrepancies from the study as planned (and, if relevant, registered) have been explained.

This is an Open Access article distributed in accordance with the Creative Commons Attribution Non Commercial (CC BY-NC 4.0) license, which permits others to distribute, remix, adapt, build upon this work non-commercially, and license their derivative works on different terms, provided the original work is properly cited and the use is non-commercial. See: http://creativecommons.org/licenses/ by-nc/4.0/

1 AAMC. Table 1: Medical students, selected years, 1965-2013. 2014 www.aamc.org/download/411782/data/2014 table1.pdf.

2 Tsugawa Y, Jena AB, Figueroa JF, Orav EJ, Blumenthal DM, Jha AK. Comparison of hospital mortality and readmission rates for medicare patients treated by male vs female physicians. JAMA Intern Med 2017:177:206-13. doi:10.1001/jamainternmed.2016.7875

3 AAMC. Matriculating student questionnaire: 2017 all schools summary report. 2017. www.aamc.org/download/485324/data/ msq2017 report.pdf.

4 Rabinowitz LG. Recognizing blind spots-A remedy for gende bias in medicine? N Engl J Med 2018;378:2253-5. doi:10.1056 NEJMp1802228

5 Files JA, Mayer AP, Ko MG, et al. Speaker introductions at internal medicine grand rounds: forms of address reveal gender bias. J Womens Health (Larchmt) 2017;26:413-9. doi:10.1089/ iwh.2016.6044

6 Jagsi R, Griffith KA, Stewart A, Sambuco D, DeCastro R, Ubel PA. Gender differences in salary in a recent cohort of early-career physician-researchers. Acad Med 2013;88:1689-99. doi:10.1097/ ACM.0b013e3182a71519

7 Jagsi R, Griffith KA, Stewart A, Sambuco D, DeCastro R, Ubel PA. Gender differences in the salaries of physician researchers. IAMA 2012;307:2410-7. doi:10.1001/jama.2012.6183

8 Seabury SA, Chandra A, Jena AB. Trends in the earnings of male and female health care professionals in the United States, 1987 to 2010. IAMA Intern Med 2013:173:1748-50.

9 Jagsi R, Griffith KA, Jones R, Perumalswami CR, Ubel P, Stewart A. Sexual harassment and discrimination experiences of academic medical faculty. JAMA 2016;315:2120-1. doi:10.1001/ jama.2016.2188

10 Adesoye T, Mangurian C, Choo EK, Girgis C, Sabry-Elnaggar H, Linos E, Physician Moms Group Study Group. Perceived discrimination experienced by physician mothers and desired workplace changes: A cross-sectional survey. JAMA Intern Med 2017;177:1033-6. doi:10.1001/jamainternmed.2017.1394

11 AAMC. The state of women in academic medicine: the pipeline and pathways to leadership, 2015-2016. 2016. www.aamc.org/ members/gwims/statistics/.

12 Guille C, Frank E, Zhao Z, et al. Work-family conflict and the sex difference in depression among training physicians. JAMA Intern Med 2017;177:1766-72. doi:10.1001/jamainternmed.2017.5138

13 Stentz NC, Griffith KA, Perkins E, Jones RD, Jagsi R. Fertility and childbearing among American female physicians. I Womens Health (Larchmt) 2016;25:1059-65. doi:10.1089/jwh.2015.5638

14 Scully RE, Davids JS, Melnitchouk N. Impact of procedural specialty on maternity leave and career satisfaction among female physicians. Ann Surg 2017;266:210-7. doi:10.1097/ SLA.0000000000002196

15 Strauss A. Basics of qualitative research: Grounded theory procedures and techniques. Sage Publications, 1990.

16 Miles M, Huberman A, Saldana J. Qualitative data analysis: an expanded sourcebook. 3rd ed. Sage Publications, 2014.

17 AAMC. 2014 Physician specialty data book. 2014. www.aamc.org/ download/473260/data/2014physicianspecialtydatabook.pdf.

18 Foschi M. Double standards for competence: Theory and research. Annu Rev Sociol 2000;26:21-42. doi:10.1146/annurev.soc.26.1.21

19 Correll S), Benard S, Paik I. Getting a job: Is there a motherhood penalty? Am / Sociol 2007;112:1297-339. doi:10.1086/511799

20 Cuddy AIC, Fiske ST, Glick P. When professionals become mothers, warmth doesn't cut the ice. / Soc Issues 2004;60:701-18. doi:10.1111/j.0022-4537.2004.00381.x

21 Heilman ME, Okimoto TG. Motherhood: a potential source of bias in employment decisions. J Appl Psychol 2008;93:189-98. doi:10.1037/0021-9010.93.1.189

22 West C, Zimmerman D. Doing gender. Gend Soc 1987;1:125-51. doi: $10.1177 / 0891243287001002002$

23 Rosaldo M, Lamphere L. Woman, culture and society. Stanford University Press, 1974

24 Eagly A. Sex differences in social behavior: A social-role interpretation. Lawrence Erlbaum, 1987.

25 Heilman ME. Description and prescription: How gender stereotypes prevent women's ascent up the organizational ladder. / Soc Issues 2001;57:657-74. doi:10.1111/0022-4537.00234

26 Benard S, Paik I, Correll S). Cognitive bias and the motherhood penalty. Hastings Law / 2008;59:1359-87.

27 Budig MJ, England P. The wage penalty for motherhood. Am Sociol Rev 2001;66:204-25. doi:10.2307/2657415

28 Budig MJ, Hodges MJ. Differences in disadvantage: Variation in the motherhood penalty across white women's earnings distribution. Am Sociol Rev 2010;75:705-28. doi:10.1177/0003122410381593

29 Ferriman K, Lubinski D, Benbow CP. Work preferences, life values, and personal views of top math/science graduate students and the profoundly gifted: Developmental changes and gender differences during emerging adulthood and parenthood. J Pers Soc Psychol 2009;97:517-32. doi:10.1037/a0016030

30 Cheryan S, Plaut VC. Explaining underrepresentation: A theory of precluded interest. Sex Roles 2010;63:475-88. doi:10.1007/ s11199-010-9835-x

31 Stratton TD, McLaughlin MA, Witte FM, Fosson SE, Nora LM. Does students' exposure to gender discrimination and sexual harassment in medical school affect specialty choice and residency program selection?Acad Med 2005;80:400-8. doi:10.1097/00001888 200504000-00020

32 Kricheli-Katz T. Choice, discrimination, and the motherhood penalty. Law Soc Rev 2012;46:557-87. doi:10.1111/j.15405893.2012.00506.x

33 Riano NS, Linos E, Accurso EC, et al. Paid family and childbearing leave policies at top US medical schools. JAMA 2018;319:611-4. doi:10.1001/iama.2017.19519

34 Villablanca AC, Beckett L, Nettiksimmons J, Howell LP. Caree flexibility and family-friendly policies: an $\mathrm{NIH}$-funded study to enhance women's careers in biomedical sciences. / Womens Health (Larchmt) 2011;20:1485-96. doi:10.1089/jwh.2011.2737

35 Blau FD, Kahn LM. Female labor supply: Why is the United States falling behind? Am Econ Rev 2013;103:251-6. doi:10.1257/ aer.103.3.251

36 Carey C. Securing the future anaesthetic workforce. Best Pract Res Clin Anaesthesiol 2018;32:25-37. doi:10.1016/j.bpa.2018.04.002

37 Budig MJ, Misra J, Boeckmann I. Work-family policy tradeoffs for mothers? Unpacking the cross-national variation in motherhood earnings penalties. Work Occup 2015;43:119-77. doi:10.1177/0730888415615385

38 James KM, Ziegenfuss JY, Tilburt JC, Harris AM, Beebe TJ. Getting physicians to respond: the impact of incentive type and timing on physician survey response rates. Health Serv Res 2011;46:232-42. doi:10.1111/j.1475-6773.2010.01181.x 